\title{
Rates of elastic compression stockings prescription following the diagnosis of deep venous thrombosis among Canadian emergency physicians and trainees
}

\author{
Ahmed Kayssi, MD, MSc, MPH*; Andrew Petrosoniak, $\mathrm{MD}^{\dagger}$; Jeremy Levenstadt, $\mathrm{MD}^{\ddagger}$; \\ Naomi Eisenberg, PT, MEd*; Sue Jenkins, $\mathrm{RN}^{\S}$; Graham Roche-Nagle, MD, MBA*
}

\section{ABSTRACT}

Introduction: Postthrombotic syndrome (PTS) is a complication of deep vein thrombosis (DVT) characterized by chronic pain, swelling, and heaviness, and may result in ulceration. Elastic compression stockings (ECS) worn daily after DVT have been shown to reduce the incidence and severity of PTS. The aim of our study was to investigate practices and perceptions of physicians regarding adjunct therapies to anticoagulation in patients diagnosed with lower extremity DVT.

Methods: A national online survey was conducted of Canadian emergency medicine staff physicians and residents ( $n=471)$ to investigate their attitudes toward the prescription of ECS post-diagnosis of DVT. A paper survey of patients in a thrombosis clinic $(n=58)$ was also administered to better understand the patient experiences with ECS.

Results: The majority of staff physician (62\%) and resident $(69 \%)$ respondents were unsure of whether ECS were effective in preventing PTS and managing venous symptoms. Only $6 \%$ of staff physicians and $7 \%$ of residents routinely prescribed ECS for above-knee DVTs. More than $78 \%$ of respondents were unsure about the optimal timing of initiation of ECS and duration of therapy. Although all patients noted symptomatic relief with ECS, only $50 \%$ were prescribed stockings by an emergency or family doctor, and $69 \%$ of those patients wore the stockings on a daily basis. Staff physicians most frequently identified poor fit as the reason for lack of patient compliance, whereas patients most frequently cited cost.

Conclusions: Our findings suggest that there is variability in practice among Canadian emergency medicine physicians and trainees and a need for widespread education regarding the latest evidence of the benefit of ECS after DVT.

Keywords: Venous Disease, Deep Vein Thrombosis, Elastic Compression Stockings, Post-thrombotic Syndrome,

Knowledge Translation, Medical Education

\section{INTRODUCTION}

Approximately $20 \%-50 \%$ of adults will develop postthrombotic syndrome (PTS) in the first 2 years after a diagnosis of deep vein thrombosis (DVT). ${ }^{1}$ PTS is characterized by swelling, venous eczema, pigmentation, severe edema, venous claudication, chronic pain, and recurrent ulcers. ${ }^{2}$ PTS is also associated with a significant health care cost, loss of economic productivity, and a deterioration in quality of life. ${ }^{3,4}$ Risk factors for PTS include recurrent DVT, obesity (body mass index greater than 30), older age, female gender, and residual DVT symptoms during 1 month after a diagnosis of DVT. ${ }^{5}$

The use of graduated elastic compression stockings (ECS) has been shown to significantly decrease the odds of developing PTS in post-DVT patients. ${ }^{6}$ Despite practice guidelines published by the American College of Physicians, the American College of Chest Physicians, and the American Academy of Family Physicians recommending the use of ECS in post-DVT patients, ${ }^{7,8}$ there is variability in reported ECS prescription patterns. Surveys of thrombosis specialists involved in the care of post-DVT patients showed $98 \%$ prescription rates in Germany and 26\% in Canada. ${ }^{9,10}$ A recent survey of Irish primary-care providers revealed a $63 \%$ prescription rate. ${ }^{11}$ Of note, there are currently no studies in the literature examining such practice patterns among emergency physicians.

The aim of this study was to assess current ECS prescription practices among Canadian emergency

From the *Division of Vascular Surgery, University of Toronto, ON; †Division of Emergency Medicine, University of Toronto, ON; $\neq D e p a r t m e n t ~ o f$ Family and Community Medicine, University of Toronto, ON; and §Thrombosis Clinic, Toronto General Hospital, University Health Network, University of Toronto, ON.

Correspondence to: Dr. Graham Roche-Nagle, Toronto General Hospital, University Health Network, 200 Elizabeth Street, Toronto, ON M5G 2C4; Email: graham.roche-nagle@uhn.ca 
physicians and trainees involved in the care of post-DVT patients. The opinions of patients and nonthrombosis clinicians on the use of ECS were also surveyed. It was hypothesized that ECS were not consistently prescribed by emergency doctors and trainees and that there was variability in practice regarding their use in patients with DVT.

\section{METHODS}

Between September 2011 and March 2012, two surveys were conducted to assess physician and patient attitudes toward ECS after DVT. The study protocol was approved by the Ethics Review Boards of the University Health Network and St. Michael's Hospital at the University of Toronto.

\section{Physician survey}

A survey was developed (Appendix A) based on experience and a literature review to gauge current physician practices and views on ECS prescription after DVT. ${ }^{11}$ The survey items were generated using the literature and by interviewing physicians and nurse practitioners experienced in prescribing ECS. The number of items was reduced based on feedback received. To ensure content face validity and question comprehension, the survey was pretested and piloted by staff and residents in vascular surgery, family medicine, and emergency medicine, as well as by a nurse practitioner at our institution's thrombosis clinic.

An online survey was then created using a commercial survey software (www.surveymonkey.com) and administered using the Dillman Tailored Design Method. ${ }^{12}$ The survey was distributed by email through the Canadian Association of Emergency Physicians membership list, which includes emergency medicine physicians and trainees from across Canada. One reminder email was sent 2 weeks after the initial invitation email. All surveys were anonymous.

\section{Patient survey}

A paper-based questionnaire was developed from experience and a literature review to assess patient perceptions and satisfaction with ECS after DVT (Appendix B). ${ }^{11}$ All patients with a DVT diagnosis who were referred to the thrombosis clinic at University Health Network, University of Toronto, were approached to participate. Each month, approximately 50 new and returning patients were approached. Recruitment occurred from September 2011 to January 2012.

\section{Data analysis}

Descriptive statistics (e.g., means, proportions) were calculated for all data. Chi-squared test statistics were generated to compare staff and resident physician responses. Statistical significance was determined at the $p=0.05$ level. All data analyses were carried out using the statistical software package STATA, version 12 (College Station, Texas). ${ }^{13}$ Figures were generated using Microsoft Excel 2010 (Redmond, Washington). ${ }^{14}$

\section{RESULTS}

\section{Physician survey}

Four hundred seventy-one physicians participated in the survey, including 403 staff emergency physicians (56\% response rate) and 68 emergency medicine residents (59\% response rate).

Most respondents did not routinely prescribe ECS to patients with newly diagnosed above-knee (94\% total, 93\% staff, 97\% residents) or below-knee DVT (93\% total, $93 \%$ staff, $93 \%$ residents). A majority of staff and resident respondents were unsure of whether ECS could prevent PTS in newly diagnosed DVT (Figure 1). Most were also unsure about the optimal timing to initiate ECS after a new diagnosis of DVT (Figure 2) or the length of time for which ECS should be prescribed (Figure 3). Staff respondents most frequently cited poor fit, need for assistance, and cosmesis as the main reasons for lack of patient compliance with ECS. Conversely, most residents cited the need for assistance in putting on the stockings, cosmesis, and pruritus as the main reasons for lack of patient compliance. Most respondents correctly believed that ECS did not increase the risk of pulmonary embolism (PE) in newly diagnosed DVT (Figure 4) but incorrectly selected Class II (20-30 $\mathrm{mmHg})$ rather than Class III $(30-40 \mathrm{mmHg})$ as the optimal strength of ECS to prevent PTS (Figure 5). Most staff and resident respondents believed that the compliance rate with ECS in patients with newly diagnosed DVT was 25\% (Figure 6). There was no significant difference between the staff and resident answers at the $p=0.05$ level for any of the binary and categorical survey questions. 


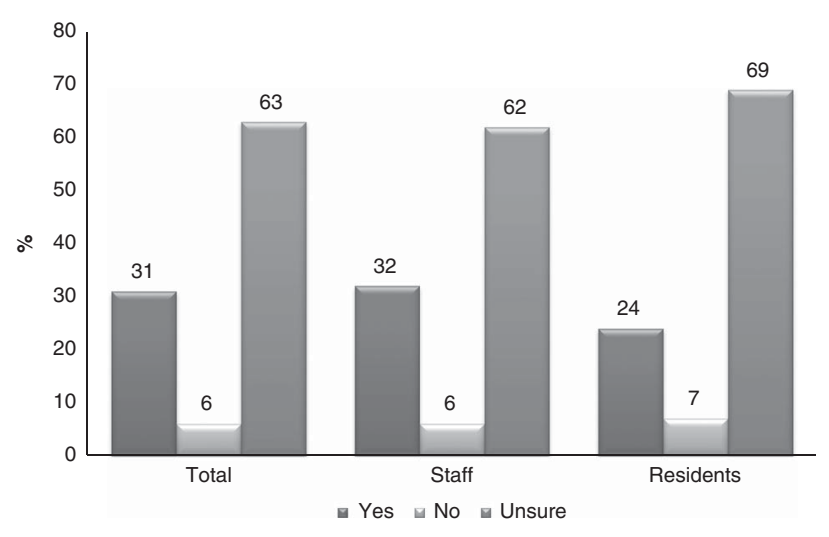

Figure 1. Can elastic compression stockings prevent postthrombotic syndrome in newly diagnosed deep vein thrombosis? Results shown as percentages of total, staff, and resident responses.

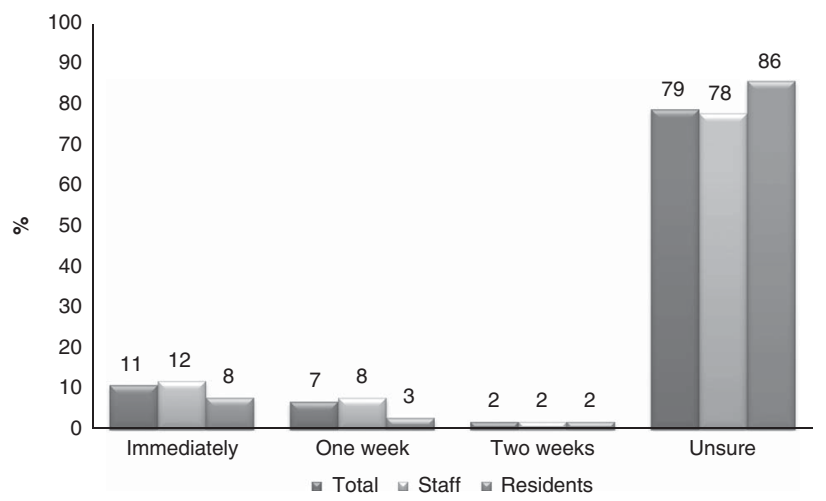

Figure 2. When is the optimal time to prescribe elastic compression stockings in newly diagnosed deep vein thrombosis? Results shown as percentages of total, staff, and resident responses.

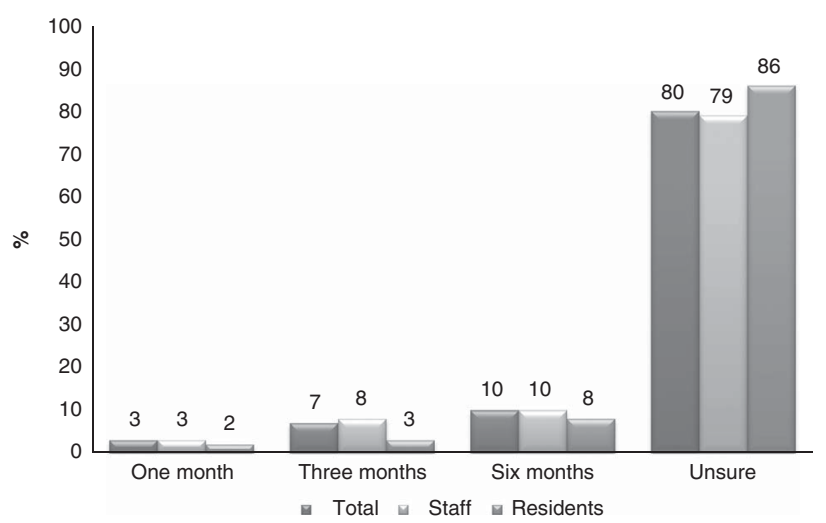

Figure 3. How long should elastic compression stockings be prescribed in newly diagnosed deep vein thrombosis? Results shown as percentages of total, staff, and resident responses.

\section{Patient survey}

Of the patients presenting to the clinic and invited to participate in this study, 58 completed the survey.

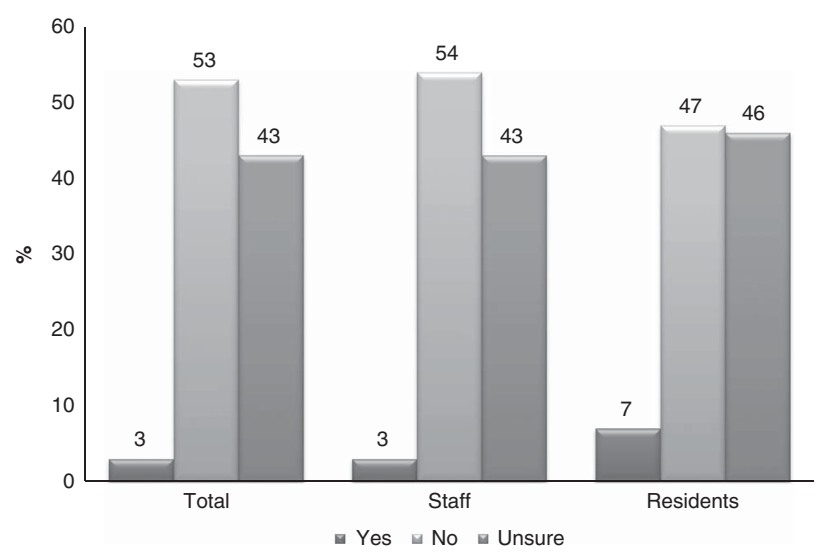

Figure 4. Do elastic compression stockings increase the risk of pulmonary embolism in newly diagnosed deep vein thrombosis? Results shown as percentages of total, staff, and resident responses.

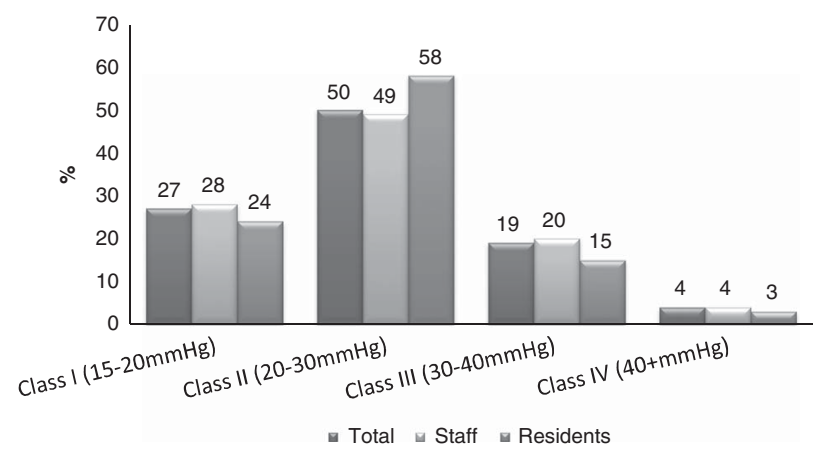

Figure 5. What is the optimal strength of elastic compression stockings to prevent postthrombotic syndrome? Results shown as percentages of total, staff, and resident responses.

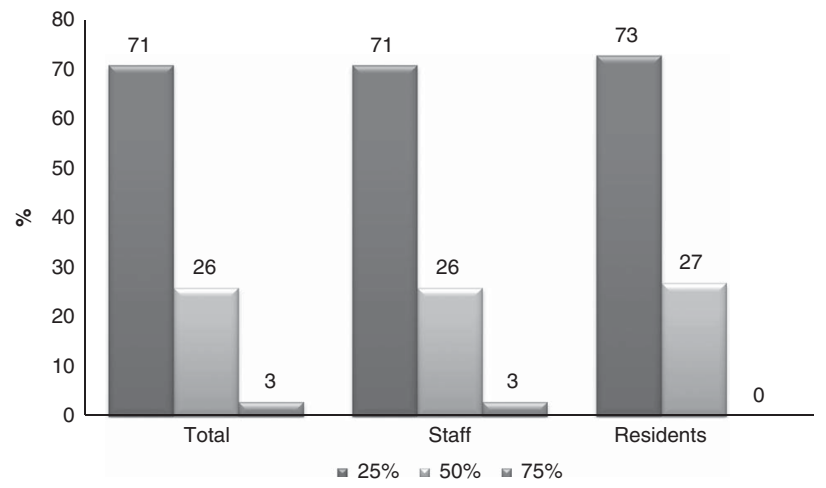

Figure 6. What is the compliance rate with elastic compression stockings in patients with newly diagnosed deep vein thrombosis? Results shown as percentages of total, staff, and resident responses.

Of those, $50 \%$ were prescribed ECS by their emergency or family medicine physician. Sixty percent of respondents subsequently bought ECS, and, of those, $69 \%$ wore them daily, whereas $20 \%$ never wore them. 
Of the patients who complied with their ECS prescriptions, all described symptomatic relief. Those who did not comply with wearing ECS most frequently listed cost, discomfort, and cosmesis as the main reasons for lack of compliance.

\section{DISCUSSION}

The aim of this study was to assess current day views and practices of Canadian emergency medicine physicians regarding the use of ECS in post-DVT patients. Our findings suggest a general lack of awareness among Canadian emergency medicine staff and trainees about the indications and uses of ECS post-DVT. Most physician respondents were unaware of when, how, and how long ECS should be prescribed.

To investigate reasons other than lack of knowledge for current ECS prescription patterns, physicians were asked if they believed that there was a relationship between ECS and a higher incidence of PE in DVT patients, which has been previously refuted. ${ }^{15}$ Most respondents did not believe that there is an association between ECS and PE.

We also investigated patient views and compliance rates with ECS. Although half of patient respondents were prescribed ECS before a referral to a thrombosis clinic for management of their DVT, only two-thirds of those patients wore them on a regular basis. These compliance rates are similar to other emergency department-prescribed outpatient treatments. Several survey-based studies have reported medication noncompliance rates between $40 \%$ and $49 \%$ upon discharge from the emergency department. ${ }^{16,17}$ The causes for lack of ECS compliance cited by patients and physicians were different. Patients were mostly concerned about cost followed by discomfort, whereas most staff physicians cited poor fit and need of assistance. A better understanding of patient needs and concerns regarding ECS might improve compliance.

Our study was limited by a relatively low response rate for staff physicians and trainees. However, systematic reviews of questionnaires have reported a $54 \%-61 \%$ mean response rate among physicians. ${ }^{18,19}$ Furthermore, Grava-Gubins and Scott reported that survey response rates among Canadian physicians and trainees varied between $28 \%$ and $32 \%$, despite employing various strategies, such as shortened surveys, emailed surveys, lottery incentives, and multiple marketing methods. ${ }^{20}$ The authors attributed a variety of factors, including survey fatigue, time pressures, and general indifference to the low response rates that they reported.

Another limitation was the recruitment of the patient respondents from a single thrombosis clinic at a tertiarycare centre with a well-established patient referral base, which likely led to selection bias. The clinic receives emergency department referrals and inpatient referrals at our institution. Evaluation for stockings, if not completed before the referral, is done by the clinic staff within $2-3$ weeks or earlier from the date that the referral is made. Nonetheless, the views of patients on lack of compliance with ECS offer a valuable perspective into the importance of adequate doctor-patient collaboration in preventing the disastrous sequelae of DVT.

In summary, we have established a substantial lack of awareness among Canadian emergency physicians and trainees regarding the indications and uses of ECS in preventing PTS. Our future work will focus on addressing this knowledge gap and developing educational initiatives to help decrease the likelihood of developing this devastating complication.

Competing interests: None declared.

\section{SUPPLEMENTARY MATERIAL}

To view supplementary material for this article, please visit http://dx.doi.org/10.1017/cem.2014.37.

\section{REFERENCES}

1. Ashrani AA, Heit JA. Incidence and cost burden of post-thrombotic syndrome. 7 Thromb Thrombolysis 2009; 28(4):465-76.

2. de Wolf MA, Wittens CH, Kahn SR. Incidence and risk factors of the post-thrombotic syndrome. Phlebology 2012; 27(Suppl 1):85-94.

3. Heit JA, Rooke TW, Silverstein MD, et al. Trends in the incidence of venous stasis syndrome and venous ulcer: a 25-year population-based study. 7 Vasc Surg 2001;33(5):1022-7.

4. Kahn SR, Hirsch A, Shrier I. Effect of postthrombotic syndrome on health-related quality of life after deep venous thrombosis. Arch Intern Med 2002;162(10):1144-8.

5. Vazquez SR, Kahn SR. Advances in the diagnosis and management of postthrombotic syndrome. Best Pract Res Clin Haematol 2012;25(3):391-402.

6. Kolbach DN, Sandbrink MW, Hamulyak K, et al. Nonpharmaceutical measures for prevention of post-thrombotic syndrome. Cochrane Database Syst Rev 2004;1:CD004174.

7. Snow V, Qaseem A, Barry P, et al. Management of venous thromboembolism: a clinical practice guideline from the American College of Physicians and the American Academy of Family Physicians. Ann Fam Med 2007;5(1):74-80. 
8. Kearon C, Akl EA, Comerota AJ, et al. Antithrombotic therapy for V'TE disease: Antithrombotic Therapy and Prevention of Thrombosis, 9th ed: American College of Chest Physicians Evidence-Based Clinical Practice Guidelines. Chest 2012;141(2 Suppl):e419S-e494S.

9. Blattler $W$, Gerlach HE. Implementation of outpatient treatment of deep-vein thrombosis in private practices in Germany. Eur 7 Vasc Endovasc Surg 2005;30(3):319-24.

10. Kahn SR, Elman E, Rodger MA, et al. Use of elastic compression stockings after deep venous thrombosis: a comparison of practices and perceptions of thrombosis physicians and patients. 7 Thromb Haemost 2003;1(3):500-6.

11. Roche-Nagle G, Ward F, Barry M. Current prescribing patterns of elastic compression stockings post-deep venous thrombosis. Phlebology 2010;25(2):72-8.

12. Dillman DA. Mail and Internet Surveys: the Tailored Design Method. New York: John Wiley and Sons, 2000.

13. StataCorp. Stata Statistical Software: Release 12. College Station, Texas: StataCorp LP, 2011.

14. Microsoft. 2003. Microsoft Excel 2010. Redmond, Washington: Microsoft.
15. Partsch $H$, Blattler W. Compression and walking versus bed rest in the treatment of proximal deep venous thrombosis with low molecular weight heparin. I Vasc Surg 2000; 32(5):861-9.

16. Hohl CM, Abu-Laban RB, Brubacher JR, et al. Adherence to emergency department discharge prescriptions. CFEM 2009;11(2):131-8.

17. Suffoletto B, Calabria J, Ross A, et al. A mobile phone text message program to measure oral antibiotic use and provide feedback on adherence to patients discharged from the emergency department. Acad Emerg Med 2012;19(8):949-58.

18. Asch DA, Jedrziewski MK, Christakis NA. Response rates to mail surveys published in medical journals. 7 Clin Epidemiol 1997;50(10):1129-36.

19. Cummings SM, Savitz LA, Konrad TR. Reported response rates to mailed physician questionnaires. Health Serv Res 2001;35(6):1347-55.

20. Grava-Gubins I, Scott S. Effects of various methodologic strategies: survey response rates among Canadian physicians and physicians-in-training. Can Fam Physician 2008;54(10): 1424-30. 